\title{
Cimicosis in Persons Previously Fed Upon by Bed Bugs
}

Johnathan M. Sheele ${ }^{1}$, Cameron Crandall ${ }^{2}$, Brandon F. Chang ${ }^{2}$, Brianna L. Arko ${ }^{3}$, Colin Dunn ${ }^{2}$, Alejandro Negrete ${ }^{2}$

1. Emergency Medicine, Mayo Clinic, Jacksonville, USA 2. Emergency Medicine, University Hospitals, Cleveland, USA 3. Clinical Research / Trauma Surgery, The MetroHealth System, Cleveland, USA

Corresponding author: Johnathan M. Sheele, sheele.johnathan@mayo.edu

\section{Abstract}

\section{Introduction}

Bed bug infestations have risen dramatically in many industrialized nations in recent decades. Most people fed upon by bed bugs will develop a pruritic rash although the frequency with which this occurs is not definitively known and may depend on host factors including the duration of the infestation.

\section{Methods}

Surveys were completed from 706 emergency department (ED) patients in Cleveland, OH about their current and past exposure with bed bugs. Subjects were asked about any post-bed bug feeding rashes that developed.

\section{Results}

There were $24 \%$ (169/698) of subjects reporting either a current or past home bed bug infestation, with 37\% (253/698) reporting they had previously been fed upon by a bed bug. Of those reporting a previous bed bug feeding, 68\% (172/253) reported a pruritic post-bed bug feeding rash and 24\% (57/237) reported developing a blister. Overall, 5\% (37/705) of ED patients reported currently having a rash, but only $2 \%$ (14/698) of ED patients reported currently have bed bugs at home and of those, only $14 \%(2 / 14)$ said they currently had a rash.

\section{Conclusion}

While $68 \%$ of ED patients reported a pruritic post-bed bug feeding pruritic rash, almost a third of persons did not report developing the rash. Post-bed bug feeding blister reactions are less common. Asking ED patients about a rash had a low sensitivity of $14 \%(2-43 \%)$ and a specificity $95 \%$ (93-96\%) to identify persons reporting home bed bugs.

Received 09/23/2019

Review began 10/09/2019 Review ended 10/14/2019 Published 10/18/2019

\section{() Copyright 2019}

Sheele et al. This is an open access article distributed under the terms of the Creative Commons Attribution License CC-BY 3.0., which permits unrestricted use, distribution, and reproduction in any medium, provided the original author and source are credited.
Categories: Dermatology, Emergency Medicine, Infectious Disease

Keywords: bedbug, bed bug, cimex lectularius, survey, rash, pruritic, pruritis, blister, prevalence, emergency department

\section{Introduction}

The common bed bug, Cimex lectularius L., is an obligate hematophagous insect that preferentially feeds on humans. Bed bugs can be one of the most common ectoparasites that clinicians encounter in industrialized nations. Within the hospital, bed bugs have been found mostly in the emergency department (ED) [1]. One hospital reported finding a bed bug within the institution every 2.2 days and within the ED every 4-5 days, resulting in a significant institutional financial burden [1-3]. ED patients with bed bugs were more likely to be older, male, arrive to the ED by ambulance, and admitted to the hospital [4-6].

Most people fed on by C. lectularius develop cimicosis, which typically manifests as a nonspecific pruritic maculopapular rash, but which can also present with bullae, vesicles, or resemble a vasculitis [7]. The prevalence of cimicosis in persons with bed bug infestations is unknown, and there are no reports on the frequency of cimicosis in hospitalized patients with bed bugs [7-8]. Cimicosis typically develops in a previously unexposed person about 10 days after the initial feeding. In sensitized individuals, the time from bed bug exposure to a reaction can be almost immediate [8]. Summaries of dermal reactions from bed bug feedings have been previously published [7-13].

The purpose of our study was to assess the frequency of self-reported pruritic and blistering cimicosis in patients reporting they had previous been fed upon by bed bugs. Additionally, we sought to determine the frequency of self-reported rashes in ED patients and assess whether this correlates with having a home bed bug infestation. 


\section{Cureus}

\section{Materials And Methods}

We received institutional review board (IRB) approval by University Hospitals to survey ED patients at a single, tertiary care, academic center in downtown Cleveland, Ohio, between June and October 2017. We surveyed 706 ED patients $\geqslant 18$ years of age who did not have psychosis, homicidal ideation, altered mentation, or dementia. Data was collected seven days a week, predominantly during day and evening hours, and we surveyed $\sim 2-3 \%$ of all ED patients during that time period. Patients reporting "unsure" or had "no answer" as responses were not included in the final data analysis. For continuous variables the mean and standard deviation (SD) were reported and analyzed using the independent t-test or analysis of variance (ANOVA). Categorical variables were summarized by frequency or percentage and analyzed using Chisquare. Binomial logistic regressions were performed using the presence of home bed bug infestations as the dependent variable. An alpha of 0.05 was set for statistical significance.

\section{Results}

Two percent of ED patients (14/698) reported having a current home bed bug infestation, and 24\% (169/698) of patients reported having either a current or past home bed bug infestation. Those with a current bed bug infestation were significantly older, 52 years (standard deviation (SD) $14 ; \mathrm{n}=14$ ) compared to those without bed bugs, 41 years $(S D 18 ; n=684)(p=0.02)$, respectively (Table 1$)[5-6]$. Regression analysis for patients reporting a current bed bug infestation and controlling for homelessness in the past year, education level, and annual income showed that age remained a significant predictor for having a current infestation (Odds ratio (OR) 1.04; 95\% confidence interval (CI) 0.009 to $0.068[p=0.01]$ ). Reporting a current home bed bug infestation was not associated with currently having a rash in the ED, insomnia, homelessness in the last year, annual income, or education level on univariate analysis $(p>0.05)$ (Table 1$)[5-6]$.

\begin{tabular}{|c|c|c|c|c|}
\hline & + bed bugs $(n=14)$ & -bed bugs $(n=684)$ & RR (95\% Cl) & $P$ value \\
\hline Mean age in years (SD) & $52(14)$ & $41(18)$ & $(-20.5$ to 1.90$)$ & 0.02 \\
\hline Current rash in the ED & & & 1.11 (0.89 to 1.37$)$ & 0.13 \\
\hline Yes & $14 \%(2 / 14)$ & $5 \%(35 / 683)$ & & \\
\hline No & $86 \%(12 / 14)$ & $95 \%(648 / 683)$ & & \\
\hline
\end{tabular}

TABLE 1: Patients reporting a current home bed bug infestation.

$\mathrm{RR}=$ relative risk; $95 \% \mathrm{Cl}=95 \%$ confidence intervals; $\mathrm{SD}=$ standard deviation; $\mathrm{ED}=$ emergency department

Thirty-seven percent of patients (253/680) reported a past history of being fed upon by a bed bug. Binomial logistic regression for ever having been fed upon by a bed bug with age as a covariate found an Odds ratio of 0.99 ( $95 \%$ CI -0.01 to 0.029 ) ( $\mathrm{p}=0.02$ ). However, when also controlling for homelessness in the past year, education level, and annual income, age was not a significant predictor (OR 0.99; 95\% CI -0.02 to 0.002 [p = 0.14]). Persons reporting previously being fed upon by a bed bug (mean: 39 years (SD 16; $\mathrm{n}=253)$ ) were younger than those who had never been fed upon by a bed bug (mean: 42 years (SD 18; $n=427))(p=0.02)$ (Table 2). This suggests that older patients are more likely to report a home bed bug infestation but younger patients were more likely to report having been fed upon by a bed bug. Lastly, there were significant differences between patients reporting a previous bed bug feeding and those who reported homelessness in the past year 56\% (24/43) compared to no homelessness 36\% $(226 / 630)(p=0.009)$. Having a current rash in the ED was not associated with ever having been fed upon by a bed bug $(\mathrm{p}=0.36)$. Sixty-nine percent $(175 / 253)$ of patients reported their last bed bug feeding was $\geqslant 12$ months ago (Table 2$)$. 


\section{Cureus}

\begin{tabular}{|c|c|c|c|c|}
\hline & Yes $(n=253)$ & No $(n=427)$ & RR (95\% Cl) & $\mathbf{P}$ \\
\hline Mean age in years (SD) & $39(16)$ & $42(18)$ & (0.56 to 5.99$)$ & 0.02 \\
\hline Homelessness in last year & & & 1.45 (1.03 to 2.04$)$ & 0.009 \\
\hline Yes & $56 \%(24 / 43)$ & $44 \%(19 / 43)$ & & \\
\hline No & $36 \%(226 / 630)$ & $64 \%(404 / 630)$ & & \\
\hline Current rash in the ED & & & $1.02(0.98$ to 1.06$)$ & 0.36 \\
\hline Yes & $6 \%(16 / 253)$ & $5 \%(20 / 426)$ & & \\
\hline No & $94 \%(237 / 253)$ & $95 \%(406 / 426)$ & & \\
\hline Pruritic cimicosis & $68 \%(171 / 235)$ & $32 \%(82 / 235)$ & & \\
\hline Mean age (SD) & $39(16)$ & $39(16)$ & (-4.00 to 4.55$)$ & 0.90 \\
\hline Blistering cimicosis & $24 \%(57 / 237)$ & $76 \%(180 / 237)$ & & \\
\hline Mean age (SD) & $41(15)$ & $38(16)$ & (-7.47 to 2.03$)$ & 0.26 \\
\hline Time from last bed bug feeding & & NA & NA & NA \\
\hline$\leq 2$ months & $8 \%(20 / 253)$ & & & \\
\hline 2-6 months & $14 \%(35 / 253)$ & & & \\
\hline 6-12 months & $9 \%(22 / 253)$ & & & \\
\hline$\geq 12$ months & $69 \%(175 / 253)$ & & & \\
\hline \multirow[t]{2}{*}{ Annual income } & & & NA & $<0.001$ \\
\hline & $71 \%(170 / 240)$ & $52 \%(212 / 406)$ & & \\
\hline$\$ 25,000-\$ 50,000$ & $25 \%(59 / 240)$ & $28 \%(114 / 406)$ & & \\
\hline$\$ 50,000-\$ 75,000$ & $3 \%(6 / 240)$ & $9 \%(38 / 406)$ & & \\
\hline$\$ 75,000-\$ 100,000$ & $1 \%(3 / 240)$ & $4 \%(17 / 406)$ & & \\
\hline$\geq \$ 100,000$ & $1 \%(2 / 240)$ & $6 \%(25 / 406)$ & & \\
\hline Education level & & & NA & $<0.001$ \\
\hline$\leq$ high school & $90 \%(226 / 252)$ & $76 \%(323 / 426)$ & & \\
\hline Associate's & $8 \%(19 / 252)$ & $9 \%(38 / 426)$ & & \\
\hline Bachelor's & $2 \%(4 / 252)$ & $10 \%(44 / 426)$ & & \\
\hline Master's & $1 \%(3 / 252)$ & $3 \%(14 / 426)$ & & \\
\hline Doctorate & $0 \%(0 / 252)$ & $2 \%(7 / 426)$ & & \\
\hline
\end{tabular}

\section{TABLE 2: Patients reporting they had previously been fed upon by a bed bug.}

$\mathrm{RR}=$ relative risk; $95 \% \mathrm{Cl}=95 \%$ confidence interval; $\mathrm{SD}=$ standard deviation

\section{Pruritic cimicosis}

Sixty-eight percent of patients (172/253) reporting a past bed bug feeding stated they developed a postfeeding dermal reaction that was pruritic. There were no significant differences $(p>0.05)$ in those patients that reported pruritic cimicosis versus no pruritis cimicosis and annual income, insomnia, having a current rash in the ED, homelessness in the last year, the time from their last known bed bug feeding, and having a current home bed bug infestation on univariate analysis. Additionally, binomial logistic regression for pruritic cimicosis when adjusting for annual income and homelessness in the last year found that age was not a significant predictor for pruritic cimicosis (OR 1.004 ; 95\% CI 0.99 to $1.02[\mathrm{p}=0.64])$. 


\section{Blistering cimicosis}

Twenty-four percent (57/237) of patients with a previous report of a bed bug feeding developed a blistering rash. Eighty-six percent (49/57) of persons reporting a previous bed bug feeding reported the bed bug feeding resulted in both pruritic and blistering cimicosis versus $14 \%(8 / 57)$ that reported blistering cimicosis without pruritis. There were no significant differences $(p>0.05)$ in those patients that reported a blistering versus non-blistering cimicosis and annual income, insomnia, homelessness in the last year, and the time from the last bed bug feeding. Binomial logistic regression for the development of blistering cimicosis when adjusting for annual income and homelessness in the last year found that age was not a significant predictor for pruritic cimicosis (OR 1.02 ; $95 \%$ CI 1.0 to 1.04 [p $=0.13]$ ).

\section{Current rash in the emergency department}

Overall, 5\% (37/705) of ED patients reported currently having a rash while in the ED. There were no significant differences for patients with and without a rash in the ED and age, level of formal education, annual income, reported insomnia, or homelessness in the past year on univariate analysis $(\mathrm{p}>0.05)$. Those patients that reported having a rash in the ED were asked to rank the degree of pruritis they were having on a scale of $1-5$ (with 1 meaning no itch and 5 meaning extreme pruritis). The mean degree of pruritis was 2.9 (SD 1.6; $\mathrm{n}=34$ ).

\section{Current rash in the emergency department and bed bugs}

On univariate analysis those patients in the ED reporting a current rash were not significantly more likely to also report a home bed bug infestation (14\% (2/14)) compared to those without bed bugs $(5 \%(35 / 682))(\mathrm{p}=$ 0.13 ); however, the low number of patients with bed bugs affects the generalizability of these findings. Using the presence of a current rash in the ED to screen for a home bed bug infestation had a sensitivity of $14 \%$ (2$43 \%$ ), specificity $95 \%$ (93-96\%), positive predictive value 5\% (2-18\%), and negative predictive value $98 \%$ (9899\%). Having a current home bed bug infestation was not associated with having a current rash in the ED using logistic regression and controlling for age, homelessness in the past year, education level, annual income (OR 1.01; 95\% CI 0.99 to 1.03 [p = 0.28]). Interestingly, 21\% (3/14) of patients with bed bugs at home reported never having been fed on by a bed bug.

\section{Discussion}

Older age was a significant predictor for having a current bed bug infestation even when controlling for potential confounding variables. On univariate analysis younger age was significantly associated with a higher chance of reporting ever having been fed upon by a bed bug, but this association was no longer significant when controlling for potential confounders. Homelessness in the past year was associated with a significant increased risk for reporting ever having been fed upon by a bed bug.

Most people reporting a previous bed bug feeding report that they developed pruritis (68\%), and fewer, reported developing a blister (24\%). Our findings are similar to those of a survey of persons with bed bugs which found that $74 \%$ developed a skin reaction to being fed up on by the insect [9]. Blistering cimicosis has been reported previously in the setting of a severe reaction to the bed bug feeding although the frequency of blistering cimicosis in the general public is unknown [14].

Current and previous exposure to bed bugs can be common among ED patients with $37 \%$ in our survey reporting that they have previously been fed upon by a bed bug. Patients with bed bugs may not be forthcoming about their infestations with their healthcare providers [5]. Bed bugs are small and seek harborage away from their human host when they are not feeding, making it harder for healthcare providers to identify an insect during a clinical encounter [2]. Diagnosing bed bug infestations can be even more challenging without a pruritic rash as was reported in $32 \%$ of subjects in our survey. Only $14 \%(2 / 14)$ ED patients reporting an active home bed bug infestation also reported having a rash in the ED, compared with $5 \%$ with a rash in the overall ED patient population. Using the presence of a current rash in the ED as a triage screening question for having an active home bed bug infestation had a low sensitivity (14\%) but high specificity (95\%), and therefore would not likely be helpful despite the high burden of bed bugs within this population.

\section{Limitations}

There are no clinical diagnostic tests for current or past exposure to bed bugs, and we were unable to confirm the presence of any bed bug exposures in our subjects. Some subjects may have felt that they were fed upon by bed bugs when in fact it may have been a different insect. Lastly, subjects may not have been able to accurately recall their past reactions to bed bugs especially since a majority of subjects reported their last bed bug feeding was $\geqslant 12$ months ago.

\section{Conclusions}

While a majority of ED patients reported a pruritic post-bed bug feeding rash, it is notable that almost a third of persons did not report developing a pruritic rash. Twenty-four percent of persons reported 
developing a post-bed bug feeding blister. The number of ED patients reporting a rash was more than twice the number that reported currently having a home bed bug infestation. Most ED patients with a home bed bug infestation did not report having a rash in the ED. Asking ED patients if they currently have a rash as a method of screening them for a reported home bed bug infestation has a low sensitivity. Results suggest that older patients are more likely to report a home bed bug infestation but younger patients were more likely to report having been fed upon by a bed bug.

\section{Additional Information \\ Disclosures}

Human subjects: Consent was obtained by all participants in this study. University Hospitals issued approval 02-17-39. This study was approved by the University Hospitals IRB. Animal subjects: All authors have confirmed that this study did not involve animal subjects or tissue. Conflicts of interest: In compliance with the ICMJE uniform disclosure form, all authors declare the following: Payment/services info: All authors have declared that no financial support was received from any organization for the submitted work. Financial relationships: All authors have declared that they have no financial relationships at present or within the previous three years with any organizations that might have an interest in the submitted work. Other relationships: All authors have declared that there are no other relationships or activities that could appear to have influenced the submitted work.

\section{Acknowledgements}

We thank Jackie Lingler for her assistance.

\section{References}

1. Sheele JM, Barrett E, Farhan O, Morris N: Analysis of bed bug (Cimex lectularius) introductions into an academic medical center. Infect Control Hosp Epidemiol. 2017, 38:623-624. 10.1017/ice.2017.13

2. Sheele JM, Barrett E, Dash D, Ridge GE: Analysis of the life stages of Cimex lectularius captured within a medical centre suggests that the true numbers of bed bug introductions are under-reported. J Hosp Infect. 2017, 97:310-312. 10.1016/j.jhin.2017.07.025

3. Totten V, Charbonneau H, Hoch W, Shah S, Sheele JM: The cost of decontaminating an ED after finding a bed bug: results from a single academic medical center. Am J Emerg Med. 2016, 34:649. 10.1016/j.ajem.2015.12.020

4. Sheele JM, Gaines S, Maurer N, et al.: A survey of patients with bed bugs in the emergency department . Am J Emerg Med. 2017, 35:697-698. 10.1016/j.ajem.2016.12.076

5. Sheele JM, Crandall CJ, Chang BF, Arko BL, Dunn CT, Negrete A: Characteristics of bed bug infested patients in the emergency department. Emerg Med Int. 2019, 2019:5. 10.1155/2019/8721829

6. Sheele JM, Crandall CJ, Chang BF, Arko BL, Dunn CT, Negrete A: Risk factors for bed bugs among urban emergency department patients. (Epub ahead of print). J Community Health. 2019, 10.1007/s10900-01900681-2

7. Goddard J, deShazo R: Bed bugs (Cimex lectularius) and clinical consequences of their bites . JAMA. 2009, 301:1358-1366. 10.1001/jama.2009.405

8. Reinhardt K, Kempke D, Naylor RA, Siva-Jothy MT: Sensitivity to bites by the bedbug, Cimex lectularius . Med Vet Entomol. 2009, 23:163-166. 10.1111/j.1365-2915.2008.00793.x

9. Potter F, Haynes KF, Connelly K, Deutsch M, Hardebeck E, Partin D, Harrison R: The sensitivity spectrum: human reactions to bed bug bites. Pest Control Technol. 2010, 38:70-74.

10. Goddard J, Edwards KT, deShazo RD: Observations on development of cutaneous lesions from bites by the common bed bug, Cimex lectularius L. Midsouth Entomol. 2011, 4:49-52.

11. Goddard J: Cutaneous reactions to bed bug bites. Skinmed. 2014, 12:141.

12. Goddard J, de Shazo R: Rapid rise in bed bug populations: the need to include them in the differential diagnosis of mysterious skin rashes. South Med J. 2008, 101:854-855. 10.1097/SMJ.0b013e31817be9db

13. Sheele JM, Ridge GE, Coppolino K, Bonfield T, Young AB, Gaines SL, McCormick TS: Antibody and cytokine levels in humans fed on by the common bedbug, Cimex lectularius L. Parasite Immunol. 2017, 39:12411. 10.1111/pim.12411

14. deShazo RD, Feldlaufer MF, Mihm MC Jr, Goddard J: Bullous reactions to bedbug bites reflect cutaneous vasculitis. Am J Med. 2012, 125:688-694. 10.1016/j.amjmed.2011.11.020 